\title{
Use of 3D mesh geometries and additive manufacturing in neutron beam experiments
}

\author{
László Szentmiklósi ${ }^{1}$ (1) · Boglárka Maróti ${ }^{1} \cdot$ Zoltán Kis $^{1} \cdot$ József Janik $^{1}$ · László Zoltán Horváth ${ }^{1}$
}

Received: 18 January 2019 / Published online: 22 March 2019

(c) The Author(s) 2019

\begin{abstract}
PGAI-NT, a neutron-based element composition and structure analysis method, is well applicable to the non-destructive characterization of valuable artefacts, paleontological, bulk geological samples or to industrial reverse engineering. To set up the measurement geometry and scanning positions for items with unconstrained shapes, sizes, and matrices, accurate knowledge of the object's geometry is a must. We applied portable structured-light 3D optical scanning or segmented neutron/X-ray tomography data to produce 3D meshes of the complex samples. Subsequently, 3D printing was used to fabricate detailed replicas of museum objects, as well as their gentle ad hoc sample holders, or produce custom parts of the Budapest PGAA instrument.
\end{abstract}

Keywords Structured-light optical scanning · Neutron/X-ray tomography · Voxel segmentation · Additive manufacturing · Replica $\cdot$ Ad hoc sample holders

\section{Introduction}

The technology required to accurately describe and replicate the geometry of an irregular-shaped object has dynamically progressed in recent years. Additive manufacturing (AM, also known as 3D printing) [1], where three-dimensional objects are directly constructed from their digital geometry representations by repeatedly depositing thin layers of a raw material, gained importance in industrial prototyping and reverse engineering [2]. The AM is no longer limited to plastic: aluminum, titanium, stainless steel, gold and silver became available as raw materials.

Scientific instruments at large-scale facilities can therefore significantly benefit from AM technology. Many components of these instruments, e.g. sample holders, sample chambers, collimators [3] or radiation shielding blocks are typically custom, where $3 \mathrm{D}$ printing can save manufacturing cost and time [4]. AM is able to realize designs that cannot be achieved with subtractive fabrication, therefore it can make constructions lighter in weight. This can be significant

László Szentmiklósi

szentmiklosi.laszlo@energia.mta.hu

1 Centre for Energy Research, Hungarian Academy of Sciences, 29-33 Konkoly-Thege Miklós street, Budapest 1121, Hungary to reduce the potential radioactivity of the near-beam structural components and the related spectroscopic background.

The beam time at such stations are expensive, so attempts have to be made to minimize the set-up time of an experiment. The alignment procedure can be shortened if the sample geometry of the object is known in advance, and e.g. during shutdown periods one can set a sequence of sample stage movements and verify that there will be no collision with the sample chamber wall. This can be achieved virtually [5] or physically. Valuable museum objects, paleontological samples, or meteorite fragments are usually not available for this exercise, as they can be borrowed only for a limited period, for the sole purpose of the experiment, and can be handled only by the curators. Tangible plastic geometrical replicas can be, however, effectively used instead by the instrument scientists, and when the real object arrives at the measurement site, a plug-and-play type sample holder will assist its rapid and unambiguous placement relative to the beam and the detection system. Moreover, if an object is measured at multiple experimental stations in a row, merging the datasets usually requires the alignment of the measurement coordinate frameworks of the different experimental stations. If one fabricates a set of disposable sample interlocks, that defines its placement relative to the object and at the same time relative to the standard sample holder of each station, the registration procedure is much simplified. 
Hereafter we present a set of examples that highlight the potential applicability of these novel technologies in our experiments carried out at the neutron beam lines of the Budapest Neutron Centre (BNC).

\section{Methodology}

For all bulky samples to be analyzed, the definition of the geometry is achieved via a surface meshes stored in STL (Standard Tessellation Language) format. This can be obtained well in advance of the neutron-beam experiment via structured-light 3D optical scanning or segmentation of 3D X-ray or neutron-tomography data.

\section{Structured-light 3D scanning}

A simple, cheap, productive and absolutely non-invasive solution has been the use of a RangeVision SMART 3D scanner [6] coupled to its TS-12 type turntable and controlled by the ScanCenter NG 2018.2 software. Once the scanner acquired a surface point cloud of 1-2 million raw points within a scanning time about $10-30 \mathrm{~min}$, the registration of the various views, data cleaning, 3D surface mesh generation, and photorealistic rendering are completed. By using the turntable, no markers are needed to be attached to the object. The optional photorealistic 3D output is an excellent feature for cultural-heritage samples to properly indicate the measurement spots on the object. Our 3D scanner is capable of providing a surface mesh with a spatial resolution of $0.12 \mathrm{~mm}$, directly comparable to the imaging performance of our neutron imaging stations [7], while the best industrial scanners can reach $10 \mu \mathrm{m}$ precision. The scanner device is fully portable, so 3D scanning can be carried out well in advance at the museum site, without the need to move the object out of the collection.

3D optical scanning has, however, limited performance if the sample is too dark, glaring, transparent, concave, heterogeneous, or has internal voids. For precious artifacts the usual 3D laser scanning anti-glare sprays (e.g. Helling 3D Anti-Glare Spray, Art. No. 119.990.001) are not applicable to improve the contrast, as they are difficult to remove from rough surfaces without a residue (risk of microscratches to the surface), and their hydrogen- and titaniumcontents would bias the element analysis results. One can bypass this problem by using a cyclododecane spray (e.g. Kremer Pigmente Art. No. 87099) instead that sublimates from an object within a few days after the application (see Fig. 1), so it does not falsify the results of a subsequent neutron-beam experiment.

\section{D imaging with neutrons or X-rays}

For objects not amenable to optical scanning, neutron or $\mathrm{X}$-ray tomography still offers a solution. X-ray imaging at the Budapest Neutron Centre can be done with a portable X-ray generator device (ERESCO MF3.1, tube voltage: 5-200-kV, max. current: $10 \mathrm{~mA}$ ) and a digital sCMOS camera (Andor Neo 5.5) focusing on the green-emitting intensifying screen (CAWO OG2). Neutron tomographic data can be taken with thermal, epithermal, fast (RAD station [8]) or cold neutrons (NORMA station [9]). Neutrons with higher kinetic energy penetrate through thicker layers of the material, but in exchange, the spatial resolution becomes worse. One has the flexibility this way to make a compromise considering the object size, penetration depth, and required spatial resolution. The RAD station has a field of view of $180 \mathrm{~mm}$, so larger objects can be imaged, while the NORMA station is best for the imaging of smaller items up to $40 \mathrm{~mm}$ in size.

The resulting voxel-based 3D dataset contains the local attenuation coefficients, a measure that allows implicit material identification. Based on this, the different constituents can be distinguished from each other as well as from the ambient air. The surface of the entire object, or its regions of interests, can be obtained using a 3D rendering

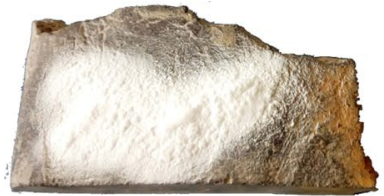

0 day

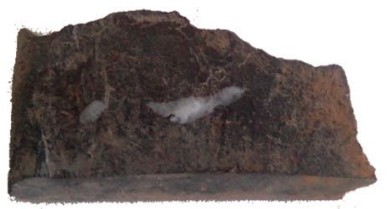

6 day

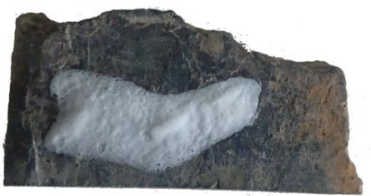

1 day

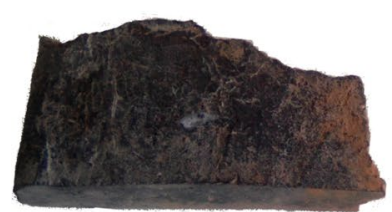

7 day

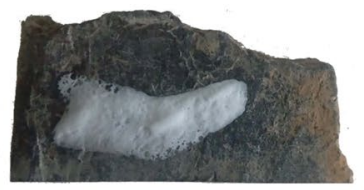

2 days

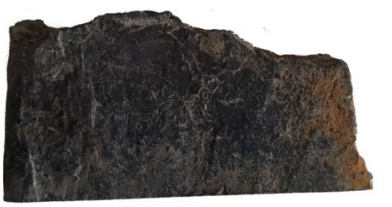

8 days

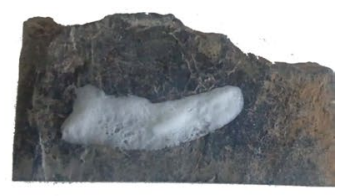

3 days

Fig. 1 The sublimation of a 1-mm-thick cyclododecane coating at an ambient temperature from a porous pottery fragment 
software (e.g. the advanced surface determination feature of the VGStudio MAX 3.2 [10]). The edge detection is based on the evaluation of the grayscale value gradient between adjacent voxels and results in a point cloud at the interfaces between various structural components and/or the air. This is converted to a geometrical model by generating a triangular surface mesh, i.e. interconnecting neighboring points to form a collection of triangles that approximate the real surface or interface. This procedure can well handle internal void regions or dishomogeneities (see the internal cavity in Fig. 2), as long as there is sufficient contrast between the regions.

\section{D printing}

A Stratasys Mojo type 3D printer [11] that makes use of the Fused Deposition Modeling (FDM) technology [2] on acrylonitrile butadiene styrene (ABS) polymer, was applied to turn the 3D data to a tangible form. When loading the STL file, care must be taken to preprocess the point cloud and make the model "watertight", i.e. the mesh must fully enclose a volume, with no gaps, holes, folds or selfintersections. The printing procedure usually takes about $1-12 \mathrm{~h}$.

\section{Results and discussion}

\section{Plastic replica of an artifact from 3D segmented data for sample stage setup}

A part of a sculpture, a South-Levantine naked goddess with palm trees and sitting monkeys on both sides (Inv. No. 2007.2-E of the Museum of Fine Arts, Budapest), is used here as a demonstration. Detailed element analysis results [12] and imaging studies [13], as well as the discussion of its cultural-heritage background, have been reported in earlier publications. As illustrated in Fig. 3, the replica, in combination with a laser pointer, can substantially assist the preparatory geometrical alignment.

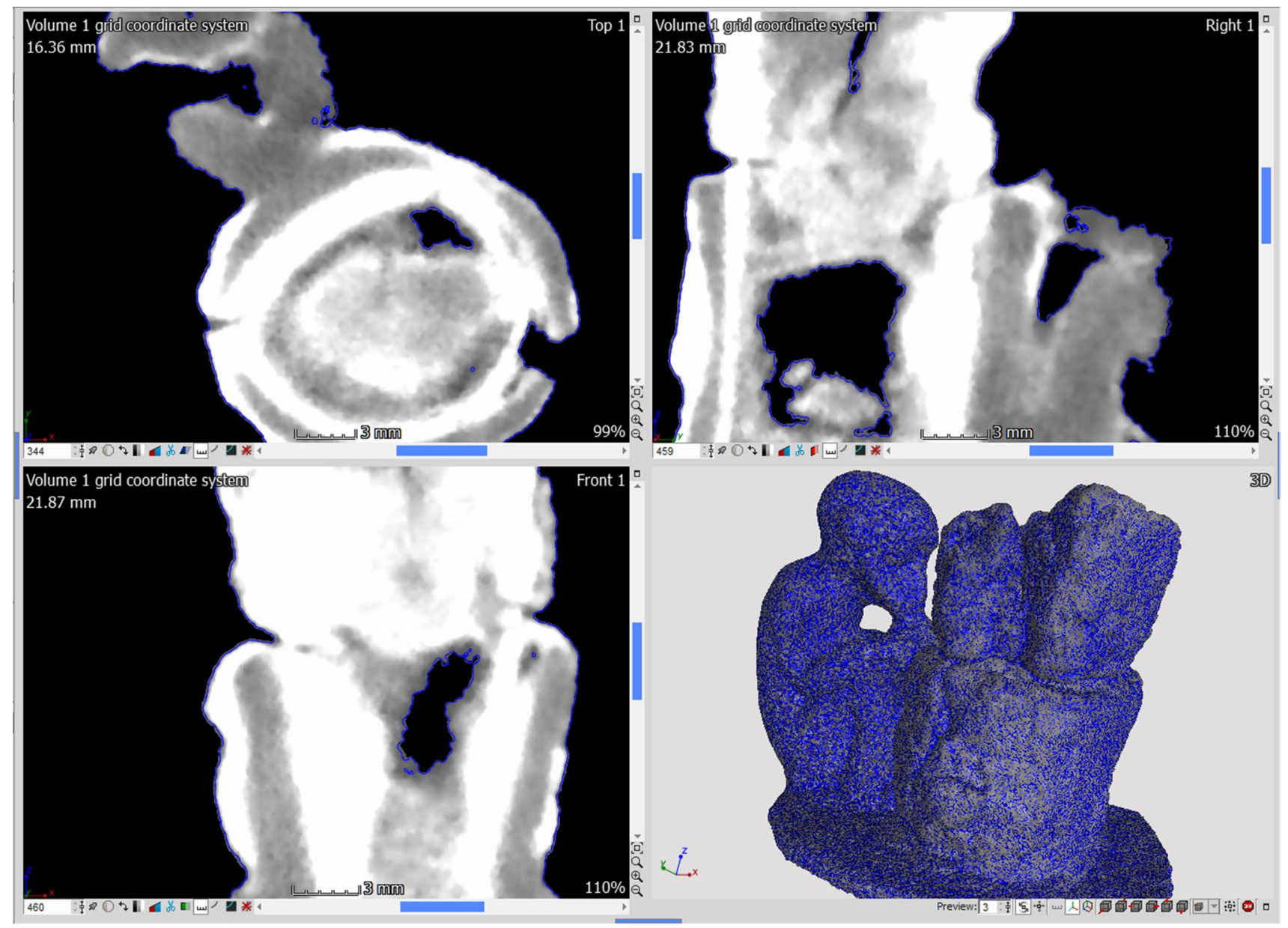

Fig. 2 The advanced surface determination on a neutron-tomography dataset (shown in white) to obtain the 3D surface mesh (shown in blue) using VGStudio MAX. Note that unlike optical scanning, the internal cavities are not part of the resulting model 
Fig. 3 a the photograph and $\mathbf{b}$ the 3D printout of the left monkey figurine from the bronze sculpture. c Replica object in the sample chamber of the NIPS-NORMA station. d-e The neutron radiograms of original and replica
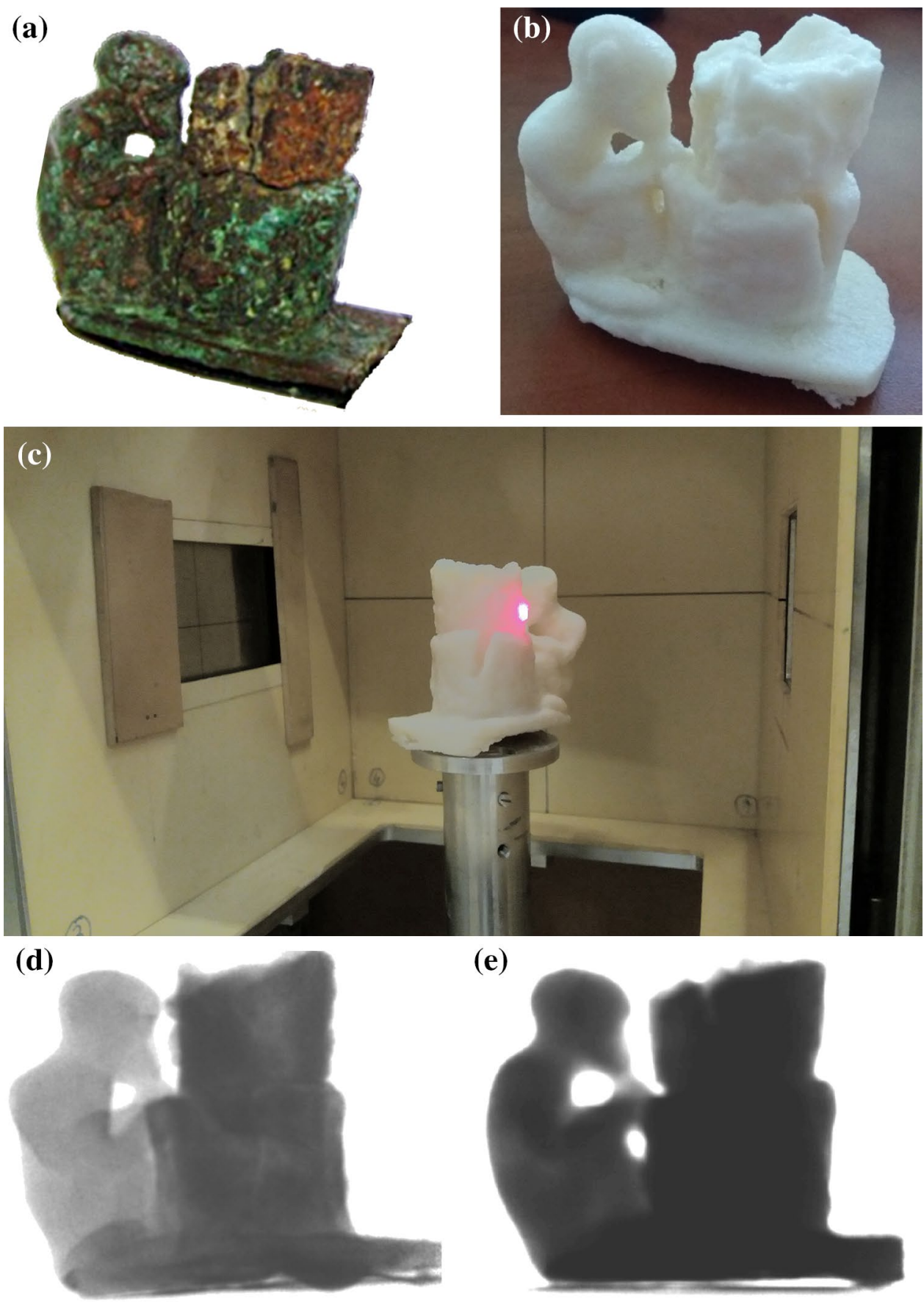

In neutron radiograms (Fig. 3d-e), one can see close agreement between the authentic and the copied object, confirming the geometrical accuracy of this approach. These plastic items, unlike the real bronze, do not get radioactive from neutron irradiation, so they are much easier to manipulate and to visually observe the placement by the experimental team. After the experiment, these 3D-printed objects can be further used to present the anatomy of a fragile object to a broad audience (e.g. open science day for students).

\section{Custom sample holders}

If an object is not solid and homogeneous, optical scanning is outperformed by segmented X-ray or neutron data. From these, the wall thicknesses not visible to the naked eye can also be derived. This allows the numerical calculation of the 
Fig. 4 a Photograph of a bronze cast spearhead. b photorealistic STL digital model. c 3D AM copy of the object. CAD concept (d) and the realization $(\mathbf{e}-\mathbf{f})$ of a custom sample interlock coupling the sample and the standard sample stage of the NIPS-NORMA station at BNC

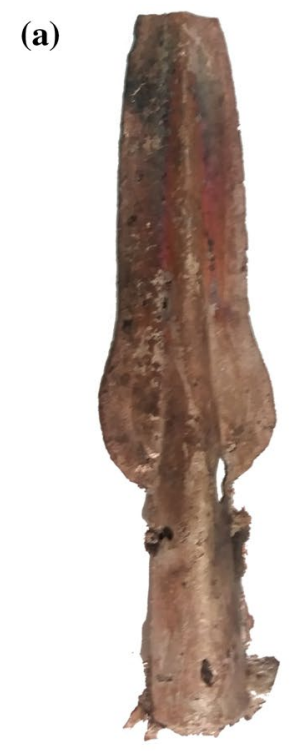

(b)

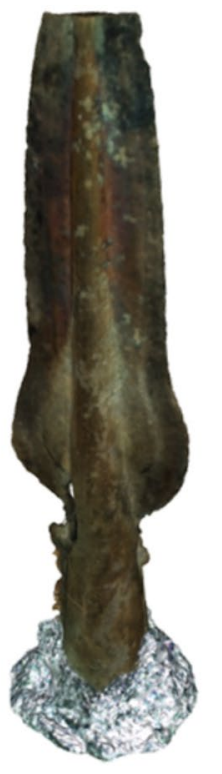

(e)

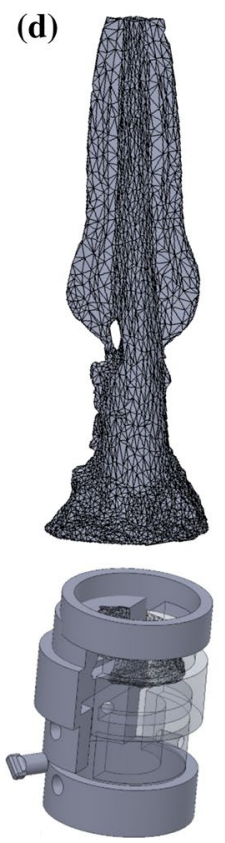

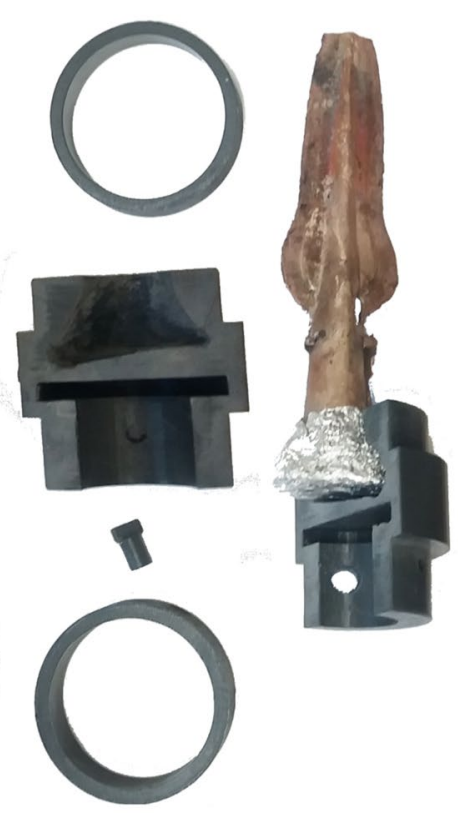

(c)

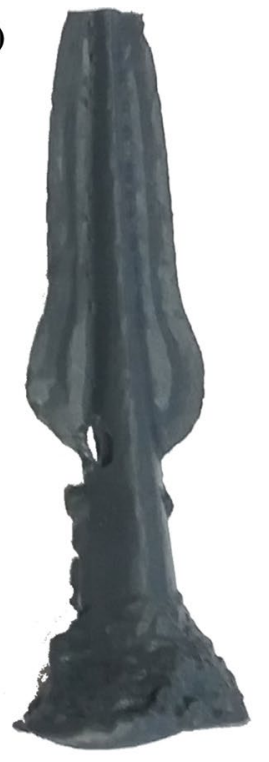

(f)

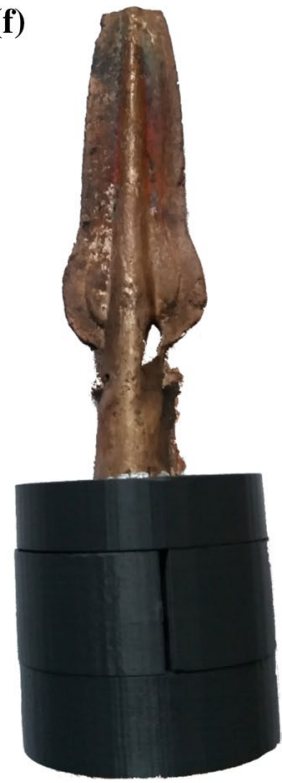

center of gravity and the principal axes. Considering this, one can fabricate an ad hoc sample holder with the AM technology that can serve as a stable, but still gentle support for the object during the neutron beam experiment (see Fig. 4). On a plastic replica one can contemplate different support options before the most suitable one will finally be applied to the valuable original object.

\section{Production of sample changer's custom components}

In prompt-gamma experiments, productivity can be enhanced and radiation dose of the instrument scientists can be lowered with increasing level of automation and remote control. A low-cost custom-made sample holder stack and the pickup mechanism for the sample frames were designed and 3D printed for use at the Prompt Gamma Activation Analysis (PGAA) facility [14, 15] of the Budapest Neutron Centre. The plastic parts (shown in blue in Fig. 5), allow great geometrical flexibility, easy assembly, and maintenance. 
Fig. 5 The sample changer of the Budapest Prompt Gamma Activation Analysis (PGAA) facility. Blue items were made with $3 \mathrm{D}$ printing
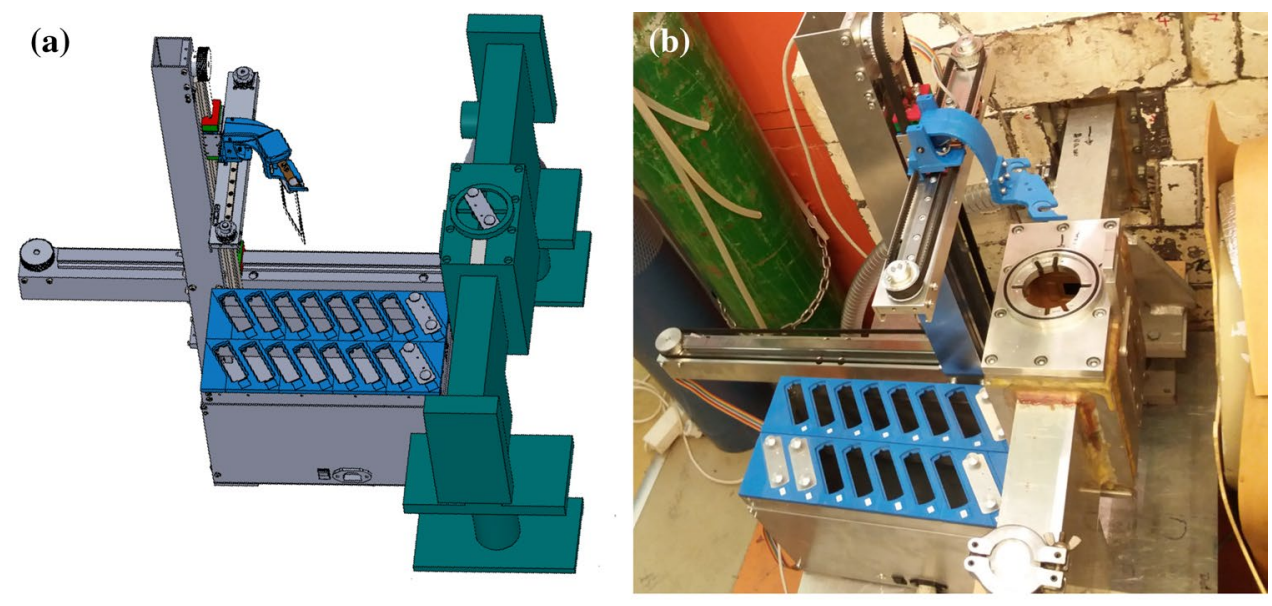

(c)

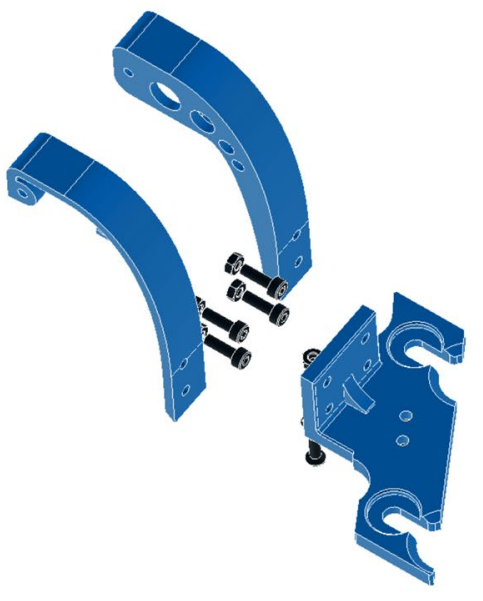

(d)

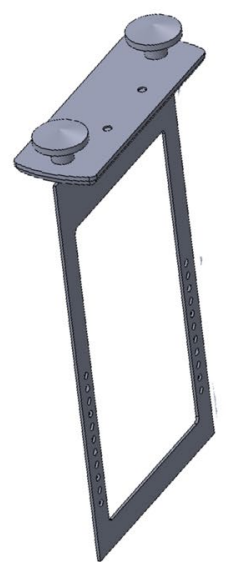

\section{Conclusions}

With the present study, it has been demonstrated that additive manufacturing, in combination with 3D geometry definition, either from structured light 3D optical scanning or segmented tomographic data, can be a significant enhancement towards the more efficient use of neutron-beam instruments. We developed a workflow based on 1:1 size replica of complex-shaped cultural heritage objects to speed up the geometrical alignment of an experiment and to securely attach the valuable samples to the facilities' sample manipulator using 3D printed sample coupling. We also used 3D printing to manufacture custom parts of an automated sample changer, in order to enhance the productivity of our PGAA facility. We expect that the application of AM will become soon a daily routine at most largescale facilities for physics worldwide.

Acknowledgements Open access funding provided by the Library of MTA Wigner Research Centre for Physics (MTA Wigner FK, MTA EK). This research was part of the Project No. 124068 that has been implemented with the support provided from the National Research,
Development and Innovation Fund of Hungary, financed under the K_17 funding scheme. L.Sz. also acknowledges the financial support of the János Bolyai Research Fellowship. We thank Thomas Calligaro of C2RMF for the fruitful discussions and the partners in WP6.3 of the IPERION CH Project (H2020 INFRAIA-1-2014-2015 Grant Agreement No. 654028) for the inspiration. We are thankful to Éva Liptay, Gábor Tarbay and Katalin Bajnok for providing the presented art objects for comprehensive 3D analysis.

Open Access This article is distributed under the terms of the Creative Commons Attribution 4.0 International License (http://creativeco mmons.org/licenses/by/4.0/), which permits unrestricted use, distribution, and reproduction in any medium, provided you give appropriate credit to the original author(s) and the source, provide a link to the Creative Commons license, and indicate if changes were made.

\section{References}

1. Attaran M (2017) The rise of 3-D printing: the advantages of additive manufacturing over traditional manufacturing. Bus Horiz 60:677-688. https://doi.org/10.1016/j.bushor.2017.05.011

2. Ngo TD, Kashani A, Imbalzano G et al (2018) Additive manufacturing (3D printing): a review of materials, methods, 
applications and challenges. Compos Part B Eng 143:172-196. https://doi.org/10.1016/j.compositesb.2018.02.012

3. Miller BW, Moore JW, Barrett $\mathrm{HH}$ et al (2011) 3D printing in $\mathrm{X}$-ray and gamma-ray imaging: a novel method for fabricating high-density imaging apertures. Nucl Instrum Methods A 659:262-268. https://doi.org/10.1016/j.nima.2011.08.051

4. Woosley S, Abuali Galehdari N, Kelkar A, Aravamudhan S (2018) Fused deposition modeling 3D printing of boron nitride composites for neutron radiation shielding. J Mater Res. https ://doi.org/10.1557/jmr.2018.316

5. James JA, Edwards L (2007) Application of robot kinematics methods to the simulation and control of neutron beam line positioning systems. Nucl Instrum Methods A 571:709-718. https://doi.org/10.1016/j.nima.2006.11.033

6. RangeVision SMART 3D scanner. https://rangevision.com/en/ products/smart/

7. Kaestner AP, Kis Z, Radebe MJ et al (2017) Samples to determine the resolution of neutron radiography and tomography. Phys Procedia 88:258-265. https://doi.org/10.1016/j.phpro.2017.06.036

8. Kis Z, Szentmiklósi L, Belgya T et al (2015) Neutron based imaging and element-mapping at the Budapest Neutron Centre. Phys Procedia 69:40-47. https://doi.org/10.1016/j.phpro.2015.07.005

9. Kis Z, Szentmiklósi L, Belgya T (2015) NIPS-NORMA station: a combined facility for neutron-based nondestructive element analysis and imaging at the Budapest Neutron Centre. Nucl Instrum Methods A 779:116-123. https://doi.org/10.1016/j. nima.2015.01.047
10. Volume Graphics VG Studio MAX 3.2. http://www.volumegrap hics.com

11. Stratasys Mojo 3D printer. http://www.stratasys.com/3d-printers/ mojo

12. Maróti B, Kis Z, Szentmiklósi L et al (2017) Characterization of a South-Levantine bronze sculpture using position-sensitive prompt gamma activation analysis and neutron imaging. J Radioanal Nucl Chem 312:367-375. https://doi.org/10.1007/s10967-017-5219-9

13. Szentmiklósi L, Maróti B, Kis Z, Kasztovszky Z (2018) Integration of neutron-based elemental analysis and imaging methods and applications to cultural heritage research. J Archaeol Sci Rep 20:476-482. https://doi.org/10.1016/j.jasrep.2018.06.001

14. Szentmiklósi L, Belgya T, Révay Z, Kis Z (2010) Upgrade of the prompt gamma activation analysis and the neutron-induced prompt gamma spectroscopy facilities at the Budapest research reactor. J Radioanal Nucl Chem 286:501-505. https://doi. org/10.1007/s10967-010-0765-4

15. Révay Z, Belgya T, Kasztovszky Z et al (2004) Cold neutron PGAA facility at Budapest. Nucl Instrum Methods B 213:385388. https://doi.org/10.1016/S0168-583X(03)01653-7

Publisher's Note Springer Nature remains neutral with regard to jurisdictional claims in published maps and institutional affiliations. 